\title{
Enhancement of Gas Permeability in HPC, CTA and PMMA under Microwave Irradiation
}

\author{
Yusuke NAKAI, Hiroaki Yoshimizu ${ }^{\dagger}$ and Yoshiharu TsujITA \\ Department of Materials Science and Engineering, Graduate School of Engineering, \\ Nagoya Institute of Technology, Gokiso-cho, Showa-ku, Nagoya 466-8555, Japan
}

(Received September 14, 2005; Accepted December 6, 2005; Published April 15, 2006)

\begin{abstract}
Gas permeability in hydroxypropyl cellulose (HPC), cellulose triacetate (CTA), and poly(methyl methacrylate) (PMMA) membranes under microwave irradiation was investigated in comparison with permeability obtained without microwave (conventional method). Permeability coefficients for several gases in HPC, CTA and PMMA under microwave were higher than those obtained by conventional methods. That is, the enhancement of gas permeability (microwave irradiation effect) by microwave was made clear, additionally it was suggested that accelerated molecular motion of $-\mathrm{OH}$ and - $\mathrm{COO}-$ moiety in side chains contribute to the enhancement under microwave irradiation. The degree of the enhancement of gas permeability depended on the combination of polymers and gases. Consequently, the size of gas molecule, polar groups and free volume spaces in polymer are assumed to affect the microwave irradiation effect. [DOI 10.1295/polymj.38.376]

KEY WORDS Gas Permeation / Microwave / Cellulose Triacetate / Hydroxypropyl Cellulose / Poly(methyl methacrylate) /
\end{abstract}

Microwave has been commonly used for radar, communication, and heating in our daily life. In particular, Microwave irradiation has been industrially applied for heating and drying of materials, and so on as one of powerful techniques of non-contact heating. ${ }^{1}$ Microwave instantaneously penetrates into heated objects and heats them from within. Utilization of microwave has been attracted attention from the aspect of green chemistry, good efficiency and so on. Utilizations of microwave on reaction of organic molecules and synthesis of polymer ${ }^{2,3}$ have been actively investigated. Specific results shown in these microwave irradiation process not seem to be unconnected with selective actions to polar functional groups by microwave.

Gas transport properties in polymers are dependent on the amount of the space through which gas molecules diffuse and the mobility of polymeric chains, ${ }^{4-9}$ that is, the high mobility of main chains and side groups leads to high gas permeability and diffusivity. Even in dense polymeric membranes, the enhancement and control of permeability due to accelerated molecular motion of polar functional groups by microwave irradiation could be expected. Reports of this type by other researchers have apparently not been published to date. In our previous study, ${ }^{10,11}$ gas permeability of cellulose acetate (CA) membranes having polar functional groups were accelerated under microwave irradiation, while gas permeability of polystyrene (PS) membranes having no polar functional groups were not affected by microwave irradia- tion. The enhancement of gas permeability for the CA membrane was considered to be due to enhanced molecular motion of polar side groups under microwave irradiation. CA contains - $\mathrm{OH}$ and $\mathrm{CH}_{3} \mathrm{COO}-$ as a polar side group. That is to say $-\mathrm{OH}$ and/or $\mathrm{CH}_{3} \mathrm{COO}-$ were suggested to contribute to the enhancement of gas permeability under microwave.

The purpose of this paper is to investigate factors governing the enhancement of gas permeability under microwave. Therefore gas permeability coefficients for cellulose triacetate (CTA), hydroxypropyl cellulose (HPC), and poly(methyl methacrylate) (PMMA) membranes were obtained under microwave irradiation and compared with the values obtained by using a conventional method.

\section{EXPERIMENTAL}

\section{Membrane Preparation}

Cellulose triacetate (CTA) and hydroxypropyl cellulose (HPC) were purchased from Aldrich Co., and Poly(methyl methacrylate) (PMMA) was supplied by Mitsubishi Rayon Co. Ltd., Japan. Sample membranes were prepared by a solution-casting method with dichloromethane for CTA, water for HPC, and chloroform for PMMA, respectively. The solutions were cast at room temperature and dried thoroughly at $30^{\circ} \mathrm{C}$ and then annealed at $120^{\circ} \mathrm{C}, 6 \mathrm{~h}$ for PMMA, $100^{\circ} \mathrm{C}$, and $12 \mathrm{~h}$ for HPC and CTA under vacuum and slowly cooled. Transparent CTA, HPC and PMMA dense membranes were obtained. In previous study, Trans-

${ }^{\dagger}$ To whom correspondence should be addressed (Tel: +81-52-735-5272, Fax: +81-52-735-5272, E-mail: yoshimizu.hiroaki@nitech.ac.jp). 
parent CA (the degree of substitute: 2.4) dense membrane was prepared by a solution-casting method from THF solution. The membranes had been stored under vacuum in a desiccator with silica gel.

\section{Characterization of Sample Membranes}

Glass transition and melting behaviour of the sample membranes were characterized using a differential scanning calorimeter (DSC), Perkin-Elmer Pyris Diamond DSC. Experiments were performed at a heating rate of $20^{\circ} \mathrm{C} / \mathrm{min}$ in nitrogen atmosphere. Glass transition temperature $\left(T_{\mathrm{g}}\right)$ was defined as a half specific heat point in the baseline shift. The melting temperature $\left(T_{\mathrm{m}}\right)$ was taken as the peak of the melting endotherm. The degree of crystallinity was evaluated from the melting enthalpy in the first scan of each sample.

Thermal gravimetric analysis (TGA) was performed using a Perkin-Elmer Pyris 1 TGA at a heating rate of $10^{\circ} \mathrm{C} / \mathrm{min}$ under $\mathrm{N}_{2}$. The amount of residual solvent and water in membranes was determined from the weight loss observed over the range $40-150^{\circ} \mathrm{C}$.

Density of sample membranes was determined by a floating method at $25^{\circ} \mathrm{C}$ using $o$-xylene and carbon tetrachloride for CTA, benzene and carbon tetrachloride for HPC, and aqueous solution of calcium nitrate for PMMA, respectively.

\section{Permeation Measurements under Microwave Irradia- tion}

Gas permeation measurements of the membranes under microwave irradiation were performed using a hand-made apparatus, which can irradiate a microwave $(2,450 \mathrm{MHz})$ to the membrane. ${ }^{10}$ The apparatus was made up by a conventional gas permeation apparatus (based on constant volume method ${ }^{12,13}$ ) and a microwave generator connected to a permeation cell through a waveguide. In a typical experiment, a membrane was set into the permeation cell. The total system, including the membrane, was exposed to vacuum to degas the membrane. Satisfactorily drying was confirmed by minimum leak rate in the downstream side. Permeant gas of $76 \mathrm{cmHg}$ was introduced on the upstream side, while the downstream pressure was relatively kept at zero. The permeation rate (the slope of the downstream pressure versus time) was monitored using a low-pressure transducer (MKS-Baratron) connected with a personal computer. After steady-state gas permeation, microwave $(2,450 \mathrm{MHz}, 500 \mathrm{~W})$ was radiated to the membrane. Permeability coefficients were evaluated from a series of data for 1800-2200s after beginning of microwave irradiation. In this time, temperature of the membrane measured by a thermometer sensor was $35^{\circ} \mathrm{C}$ because of heat generated by driving of the apparatus. Permeability coefficients under microwave irradiation were obtained for $\mathrm{He}$,
Table I. Thermal and physical properties of HPC, CA, CTA and PMMA membranes

\begin{tabular}{lllll}
\hline & HPC & CA & CTA & PMMA \\
\hline$T_{\mathrm{g}}\left({ }^{\circ} \mathrm{C}\right)$ & $7-40^{*}$ & 190 & 188 & 101 \\
$T_{\mathrm{m}}\left({ }^{\circ} \mathrm{C}\right)$ & 198 & 210 & 285 & - \\
$\chi_{\mathrm{c}}$ & 0.1 & 0.03 & 0.4 & - \\
Density $\left(\mathrm{g} / \mathrm{cm}^{3}\right)$ & 1.189 & 1.304 & 1.308 & 1.201 \\
FFV & 0.169 & 0.183 & 0.214 & 0.125 \\
Weight loss $(\%)$ & 0.1 & 0.1 & 0.2 & 0.1 \\
\hline
\end{tabular}

* References $14-17$.

$\mathrm{O}_{2}, \mathrm{Ar}, \mathrm{CO}_{2}, \mathrm{~N}_{2}, \mathrm{Kr}$ and Xe. Additionally, for the comparison purposes the permeability coefficients without microwave irradiation at $35^{\circ} \mathrm{C}$ were obtained using conventional constant volume method.

\section{RESULTS AND DISCUSSION}

\section{Characterization of Sample Membranes}

Thermal and physical properties for the sample membranes are listed in Table I. Glass transition temperature $\left(T_{\mathrm{g}}\right)$ for HPC could not clearly be observed in our DSC analysis. However, $T_{\mathrm{g}}$ of $7-40^{\circ} \mathrm{C}$ were reported as a result of DSC measurements and dynamic mechanical analyses (DMA). ${ }^{14-17}$ Therefore, $T_{\mathrm{g}}$ for HPC is approximately equal to the temperature of permeation measurement $\left(35^{\circ} \mathrm{C}\right)$. On the other hand, $T_{\mathrm{g}}$ for CA, CTA and PMMA were higher than $35^{\circ} \mathrm{C}$. Therefore, CA, CTA and PMMA are glassy state in permeation measurement.

One melting endothermic peak was observed in DSC thermograms for HPC, CA and CTA except for PMMA. The weight fraction crystallinity $\left(\chi_{c}\right)$ in the membranes was estimated as follows:

$$
\chi_{\mathrm{c}}=\frac{\Delta H}{\Delta H_{\mathrm{c}}}
$$

where $\Delta H$ is the melting enthalpy obtained from the peak area of a melting endothermic peak, and $\Delta H_{\mathrm{c}}$ is the melting enthalpy for a perfect crystallite. The value of $\Delta H_{\mathrm{c}}=6.44 \mathrm{cal} / \mathrm{g}$ for $\mathrm{HPC}^{17}$ and $8.2 \mathrm{cal} / \mathrm{g}$ for CA and $\mathrm{CTA}^{18}$ were used in this study. One exothermic peak derived from cold crystallization was appeared at about $215^{\circ} \mathrm{C}$ in DSC thermograms of CTA, so $\Delta H$ for CTA was defined as the difference calculated by subtracting the enthalpy of crystallization from the melting enthalpy. In general, gas molecules do not permeate crystal region. Additionally, microwave is supposed to have no direct effect on the crystal lattice because we confirmed permeability coefficients after microwave irradiation are equal to those before microwave irradiation. ${ }^{11}$ Consequently, it is believed that the crystal phase has no direct effect on gas permeability under microwave irradiation. 
Since water having high dielectric loss coefficient is easily heated by microwave irradiation, water sorbed in sample membranes is an unfavourable factor in this study focusing on microwave and polymers. The small amounts of residual solvent and water (0.1$0.2 \%$ ) in the membranes estimated from TGA suggests that the membranes are in a state of sufficient dehydration, so the influence of sorbed water and residual solvent on the microwave irradiation effect is negligible.

The amount of free volume in a polymer has been recognized as a major factor contributing to gas permeability and diffusivity. ${ }^{4-7}$ The fractional free volume (FFV) of amorphous regions provides indirect information about mobility of gas molecules in the polymer matrix, so FFV of the amorphous phase for each sample membrane were estimated as follows: ${ }^{5,6,19}$

$$
\mathrm{FFV}=\frac{V_{\mathrm{sp}}-1.3 V_{\mathrm{w}}}{V_{\mathrm{sp}}}
$$

where $V_{\mathrm{sp}}$ is the specific molar volume of amorphous phase, and $V_{\mathrm{w}}$ is the van der Waals volume of the repeat unit, which can be estimated by group contribution method. ${ }^{19}$ The density $\left(\rho_{\mathrm{a}}\right)$ of amorphous phase can be calculated from the experimental density $(\rho)$ data and the crystallinity $\left(\chi_{\mathrm{c}}\right)$ by assuming that the ratio of the density for crystal phase to amorphous is 1.13 as follows: ${ }^{19}$

$$
\frac{\rho_{\mathrm{a}}}{\rho}=1-\frac{0.13 \chi_{\mathrm{c}}}{1.13}
$$

Molecular chain packing of PMMA that has a FFV of 0.125 is tighter than those of other polymers in this study. On the other hand, chain packing of CTA that has a higher FFV of 0.214 is looser, that is, gas molecules can be expected to have a higher mobility.

\section{Permeability under Microwave Irradiation}

Permeability coefficients obtained in measurements with or without (conventional) microwave irradiation for the seven gases in HPC, CTA and PMMA membranes at $35^{\circ} \mathrm{C}$ are listed in Table II. Permeability co- efficients for Xe in PMMA are so small as to be unable to be obtained by our permeation apparatus. The gas permeability obtained using conventional method increase in the order PMMA $<$ HPC $<$ CTA, which is generally consistent with the order of FFV. Permeability coefficients for a number of gases under microwave irradiation are higher than those without microwave in HPC, CTA and PMMA membranes as well as the CA membrane. This result indicates the enhancement of gas permeability by microwave irradiation. Furthermore it can be considered that the enhancement of gas permeability under microwave irradiation result from accelerated molecular motions of polar functional groups, which is more active than the mobility at actual temperature $\left(35^{\circ} \mathrm{C}\right.$ in here) of the membrane. HPC has $-\mathrm{OH}$ moiety, while CTA and PMMA have-COO- moiety as a polar functional group. The enhancement of gas permeability for HPC, CTA and PMMA membranes by microwave irradiation demonstrates a contribution of $-\mathrm{OH}$ and - $\mathrm{COO}-$ to the microwave irradiation effect. Therefore it is supposed that the microwave irradiation effect in the CA membrane reported elsewhere ${ }^{10}$ is attributable to $-\mathrm{OH}$ and $\mathrm{CH}_{3} \mathrm{COO}-$ groups.

Degree of the enhancement of gas permeability depends on the kinds of polymers and gases. The relative permeability, the ratio of the permeability coefficient with microwave to that obtained by the conventional method, can be considered as a magnitude of microwave irradiation effect (the enhancement of permeability). Additionally, it can be considered that the enhanced gas permeability under microwave irradiation is attributable to the enhancement of diffusivity. Gas diffusivity in polymers has been reported the strongly correlations with gas molecular size that the logarithm of diffusion coefficients is inversely proportional to the squared effective molecular diameter, $d_{\mathrm{ef}}{ }^{2}{ }^{20,21}$ Figure 1 shows a correlation between the relative permeability and $d_{\mathrm{ef}}{ }^{2}$ for HPC with the previous result of CA. ${ }^{10}$ Figure 2 shows a correlation between the relative permeability and $d_{\mathrm{ef}}{ }^{2}$ for CTA and PMMA membranes.

For glassy polymer, some researchers have reported

Table II. Permeability coefficients in the HPC, CTA and PMMA membranes at $35^{\circ} \mathrm{C}$ with or without microwave irradiation

\begin{tabular}{llccccccc}
\hline \multirow{2}{*}{ Polymer } & \multirow{2}{*}{ Method } & \multicolumn{6}{c}{ Permeability coefficient $\times 10^{10}\left(\mathrm{~cm}^{3}(\mathrm{STP}) \mathrm{cm}^{\prime} \mathrm{cm}^{2} \mathrm{~s} \mathrm{cmHg}\right)$} \\
\cline { 3 - 8 } & & $\mathrm{He}$ & $\mathrm{O}_{2}$ & $\mathrm{Ar}$ & $\mathrm{CO}_{2}$ & $\mathrm{~N}_{2}$ & $\mathrm{Kr}$ & $\mathrm{Xe}$ \\
\hline \multirow{2}{*}{$\mathrm{HPC}$} & Microwave & 9.7 & 0.69 & 0.93 & 6.1 & 0.32 & 0.68 & 0.50 \\
& Conventional & 7.6 & 0.62 & 0.48 & 3.9 & 0.17 & 0.35 & 0.26 \\
\multirow{2}{*}{$\mathrm{CTA}$} & Microwave & 28 & 1.8 & 0.88 & 9.5 & 0.32 & 0.53 & 0.22 \\
& Conventional & 26 & 1.7 & 0.82 & 9.2 & 0.33 & 0.36 & 0.11 \\
\multirow{2}{*}{ PMMA } & Microwave & 12 & 0.31 & 0.14 & 1.1 & 0.079 & 0.066 & - \\
& Conventional & 12 & 0.23 & 0.083 & 0.78 & 0.039 & 0.022 & - \\
\hline
\end{tabular}




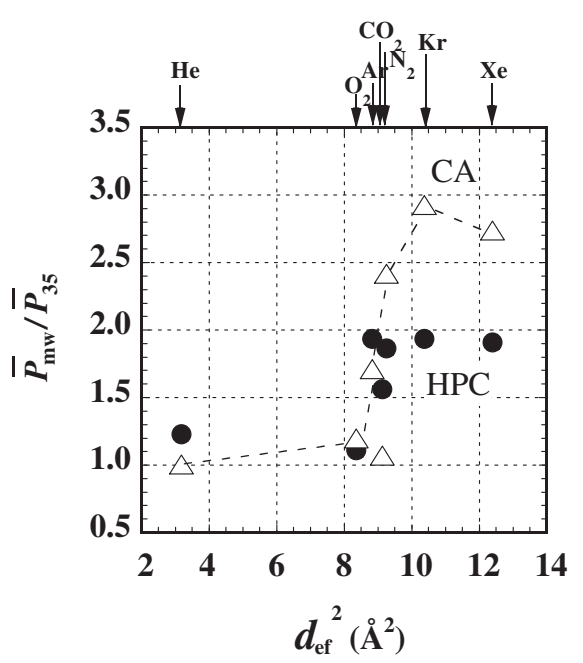

Figure 1. Correlation between relative permeability and gas molecular size for CA and HPC.

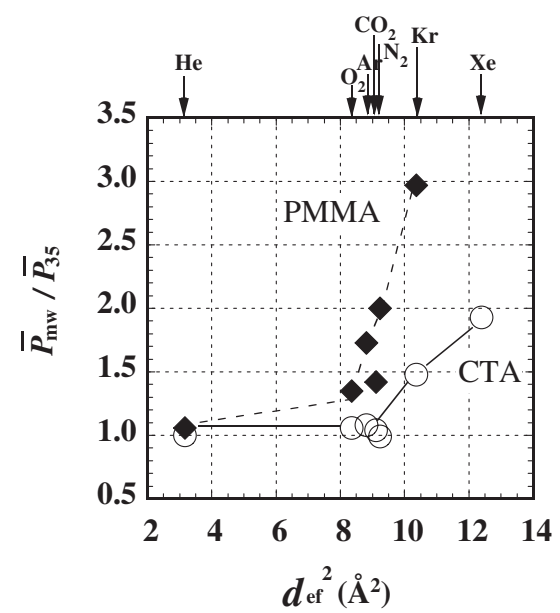

Figure 2. Correlation between relative permeability and gas molecular size for CTA and PMMA.

that sub- $T_{\mathrm{g}}$ relaxations involved in a local chain motion and/or side chain motion, which be investigated by the dynamic mechanical analysis and so on, correlate with gas permeability, ${ }^{6,8,9}$ that is, small scale local motions such as local and side chain motion as well as FFV affects gas permeability and/or diffusivity. The enhanced gas permeability under microwave is assumed to be due to the accelerated molecular motion that produce excess diffusion path. In such cases, the degree of enhancement and its dependence on the gas molecular size demonstrated in Figures 1 and 2 are thought to depend on the kinds of polar groups influenced by microwave and the amount of free volume in a polymer, which the kinds of polar groups probably determines the types of molecular motion under microwave and the FFV represents the size of the space through which gas molecules diffuse, additionally, even the same motion in the same space (i.e. same polymer) give the different effect to each gas that has different size because the size of available space to diffusion depends on the size of gas.

As compared with the CA membrane showing the most remarkable microwave irradiation effect at $\mathrm{Kr}$, the enhancement of permeability for HPC under microwave is relatively low, moreover there are no great differences in the relative permeability among the sizes of gas molecules for the HPC membrane. $\mathrm{HPC}$ were in glass transition range at $35^{\circ} \mathrm{C}$ (experimental temperature), that is, micro-Brownian motions begin to occur. Microwave irradiation is believed to induce locally enhanced molecular motions of polar functional groups attached to the polymer backbone. Thus the effect of small-scale motions enhanced by microwave on the gas permeability is thought to be relatively low at around $T_{\mathrm{g}}$ because great chain motions such as micro-Brownian motions contribute the gas permeability and reduce the impact of small-scale motions enhanced by microwave. As a result, the enhanced permeability and its dependence on gas molecular size for HPC membranes, which is in glass transition range, is generally lower than CA membranes.

It is assumed that the small-scale motion accelerated by microwave is limited space and local motion at molecular level, and specified diffusion path extended by the small-scale motion has an advantage in diffusion of specified gas molecular size as was pointed out in the previous paper. ${ }^{10}$ Then, microwave irradiation is considered to have a small effect on permeability for too large or small gas molecules relative to the specified diffusion path. For PMMA, the relative permeability of He indicates 1 signifying lack of the enhancement in permeability. Additionally the relative permeability increases with $d_{\mathrm{ef}}{ }^{2}$ from $\mathrm{O}_{2}$ to $\mathrm{Kr}$. For CTA, the permeability for $\mathrm{He}, \mathrm{O}_{2}, \mathrm{Ar}, \mathrm{CO}_{2}$ and $\mathrm{N}_{2}$ has little enhancement, on the other hand, the enhancement of permeability for $\mathrm{Xe}$ is higher than that for $\mathrm{Kr}$. There is a similarity between microwave irradiation effect of PMMA and CTA membranes. In other words, the larger gas molecule tends to show the higher enhancement of permeability derived from microwave irradiation. It is thought that the same -COO- moiety results in similarity between the enhanced permeability of CTA and PMMA, the local motion of dipoles induced by microwave irradiation might locally generate relatively large space that contributes to diffusion of relatively large gas molecules.

Gas molecular size at which the relative permeability increases and the magnitude of relative permeability should depend on polymer properties such as the kinds of polar groups and free volume space. The microwave irradiation effect is larger and occurs at smaller gas molecule in PMMA relative to CTA. Furthermore the FFV of PMMA is lower than that of CTA, that is, gas molecules are in small space and 
lower mobility state in PMMA. Therefore, It can be considered that the enhancement in diffusion for gas molecules having lower mobility is larger, because accelerated small-scale motions under microwave have a larger impact on diffusivity in close space consisting of smaller FFV and larger gas molecules.

\section{CONCLUSIONS}

Microwave irradiation results in the enhancement of permeability for various gases in HPC, CTA and PMMA, which the enhancement cannot be obtained by the usual thermal motion of overall polymer molecules with increasing temperature. As a result, it is suggested that $-\mathrm{OH}$ and $-\mathrm{COO}-$ moiety in polymers contribute to the enhancement of gas permeability under microwave. The degree of the permeability enhancement depends on the combination of polymers and gases, that is, relatively low enhancements and its poor dependences on the size of gas molecules are observed in HPC, additionally, the enhancement in CTA and PMMA are larger for large gas molecule, the enhancement in PMMA is greater than that in CTA. These results suggest that the enhanced gas permeability caused by accelerated small-scale molecular motion depends on the size of gas molecule, the kinds of polar groups and free volume space in polymer.

\section{REFERENCES}

1. A. C. Metaxas and R. J. Meredith, "Industrial Microwave Heating,” Peter Peregrinus Ltd., London U.K., 1983.

2. M. Iannelli, V. Alupei, and H. Ritter, Tetrahedron, 61, 1509 (2005).

3. H. Yeganeh, B. Tamami, and I. Ghazi, Eur. Polym. J., 40,
2059 (2004).

4. Y. Osada, T. Nakagawa, "Membrane Science and Technology,” Marcel Dekker, Inc., New York, 1992.

5. A. Thran, G. Kroll, and F. Faupel, J. Polym. Sci., Part B: Polym. Phys., 37, 3344 (1999).

6. J. S. McHattie, W. J. Koros, and D. R. Paul, Polymer, 33, 1701 (1992).

7. K. Haraya and S.-T. Hwang, J. Membr. Sci., 71, 13 (1992).

8. S. Singla, H. W. Beckham, and M. E. Rezac, J. Membr. Sci., 208, 257 (2002).

9. I.-W. Kim, K. J. Lee, J. Y. Jho, H. C. Park, J. Won, Y. S. Kang, M. D. Guiver, G. P. Robertson, and Y. Dai, Macromolecules, 34, 2908 (2001).

10. Y. Nakai, H. Yoshimizu, and Y. Tsujita, J. Membr. Sci., 256, 72 (2005).

11. Y. Tsujita, Y. Nakai, H. Yoshimizu, and M. Yamauchi, in "Advanced Materials for Membrane Separations, ACS Symposium Series 876," chapt. 20, I. Pinnau and B. D. Freeman, Ed., American Chemical Society, Washington, D.C., 2004, p 300.

12. T. T. Moore, S. Damle, P. J. Williams, and W. J. Koros, J. Membr. Sci., 245, 227 (2004).

13. W. J. Koros, D. R. Paul, and A. A. Rocha, J. Polym. Sci., Polym. Phys. Ed., 14, 687 (1976).

14. E. Marsano, E. Bianchi, S. Gagliardi, and F. Ghioni, Polymer, 41, 533 (2000).

15. S. Suto and T. Kobayashi, J. Appl. Polym. Sci., 70, 1465 (1998).

16. S. Yano, Polymer, 35, 5565 (1994).

17. T. G. Rials and W. G. Glasser, J. Appl. Polym. Sci., 36, 749 (1988).

18. A. C. Puleo and D. R. Paul, J. Membr. Sci., 47, 301 (1989).

19. D. W. van Krevelen, "Properties of Polymers," 3rd ed., Elsevier, Amsterdam, 1990.

20. V. Teplyakov and P. Meares, Gas Sep. Purif., 4, 66 (1990).

21. J. R. Fried and P. Ren, Comput. Theor. Polym. Sci., 10, 447 (2000). 